\title{
Optical determination of flexoelectric coefficients and surface polarization in a hybrid aligned nematic cell
}

\author{
A. Mazzulla* and F. Ciuchi \\ Unità INFM di Cosenza, Dipartimento di Fisica, Università della Calabria, Rende, Italy \\ J. R. Sambles \\ Thin Film Photonics Group, School of Physics, Exeter University, Exeter, Great Britain
}

(Received 18 December 2000; published 24 July 2001)

\begin{abstract}
We present an optical study of the influence of both the flexoelectric effect and surface polarization on a hybrid-aligned nematic cell using the half-leaky guided mode technique. Tilt angle profiles, obtained from fits of experimental data (reflectivity curves) taken under applied voltages, are compared with the ones derived by a complete theoretical model. Measurements with an applied alternating voltage allow the evaluation of the anchoring energy by solving the torque balance equation at the planar surface. From measurements with static fields, the sum of flexoelectric coefficients and the surface polarization are determined by numerical solution of Euler-Lagrange equations.
\end{abstract}

DOI: 10.1103/PhysRevE.64.021708

PACS number(s): 61.30.Gd, 42.70.Df

\section{INTRODUCTION}

Knowledge of the director distribution inside a liquidcrystal cell is very important since it determines several physical characteristics of the cell itself and also because it is strongly affected by external fields. Generally, typical optical techniques, such as polarized light transmission, give only integrated information on the director distribution through the cell. Therefore, the final results for the director profiles are strongly dependent on the assumed model; many different director profiles are able to give the same optical response. In contrast, reflectivity curves from the liquid-crystal sample observed using the half-leaky guided mode (HLGM) technique contain information on the detailed director structure through the cell, allowing the full director profile to be established.

Here the flexoelectric coefficients [1] and the surface polarization of a liquid-crystal cell are determined making use of this HLGM procedure. Many attempts have been made so far to measure these quantities, but several difficulties have been encountered. One of the main problems has been the inability to separate clearly the different polar terms. Electrochemical reaction of the electrodes, due to the applied dc voltage, may also be significant, in addition to such effects as ionic screening and charge accumulation. For these reasons, the experimental values of flexoelectric coefficients and surface polarization obtained by different authors using various techniques tend to disagree, even in sign [2-9].

\section{THEORY}

\section{ac field}

Taking the $z$ axis as the direction perpendicular to the cell surfaces and $\theta$ the angle between the normal and the liquid-

\footnotetext{
*Author to whom correspondence should be addressed. Email address: Mazzulla@fis.unical.it
}

crystal director, $\mathbf{n}=(\sin \theta \cos \phi, \sin \theta \sin \phi, \cos \theta)$, the Gibbs free energy per unit area of the cell is

$$
F_{G}=\int_{0}^{d} f_{G} d z=\int_{0}^{d}\left(f_{s}-f_{e}\right) d z,
$$

where $f_{G}$ is the Gibbs free-energy density, that is, the difference between strain and electrostatic density, given, respectively, by [10]

$$
\begin{gathered}
f_{s}=\frac{1}{2} f(\theta)\left(\frac{d \theta}{d z}\right)^{2}, \\
f_{e}=\frac{1}{2}\left[\left(\varepsilon_{\|} \cos ^{2} \theta+\varepsilon_{\perp} \sin ^{2} \theta\right) E^{2}\right] .
\end{gathered}
$$

Here $f(\theta)=k_{11} \sin ^{2} \theta+k_{33} \cos ^{2} \theta, \varepsilon_{\|}$and $\varepsilon_{\perp}$ are the dielectric tensor components of the liquid crystal (LC), and $k_{11}, k_{33}$ are the splay and bend elastic constants.

Applying the Euler-Lagrange equation under the constant applied voltage condition [11], we have the two differential equations

$$
\begin{gathered}
-\left(\frac{d V}{d z}\right)\left(\varepsilon_{\|} \cos ^{2} \theta+\varepsilon_{\perp} \sin ^{2} \theta\right)=D_{z}=C, \\
f(\theta)\left(\frac{d \theta}{d z}\right)^{2}-\frac{D_{z}^{2}}{\left(\varepsilon_{\|} \cos ^{2} \theta+\varepsilon_{\perp} \sin ^{2} \theta\right)}=A,
\end{gathered}
$$

where $D_{z}$ is the $z$ component of the dielectric displacement and $A, C$ are integration constants. Following the same procedure as in [10], Eqs. (4) and (5) have been converted into integral equations by using the boundary conditions, and the model director distribution inside the liquid crystal has been determined; no twist is considered.

\section{dc field}

Instead of Eq. (3), in this case we have to take the electrostatic energy density as 
(a)

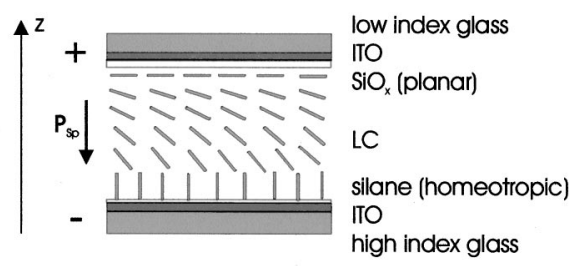

(b)

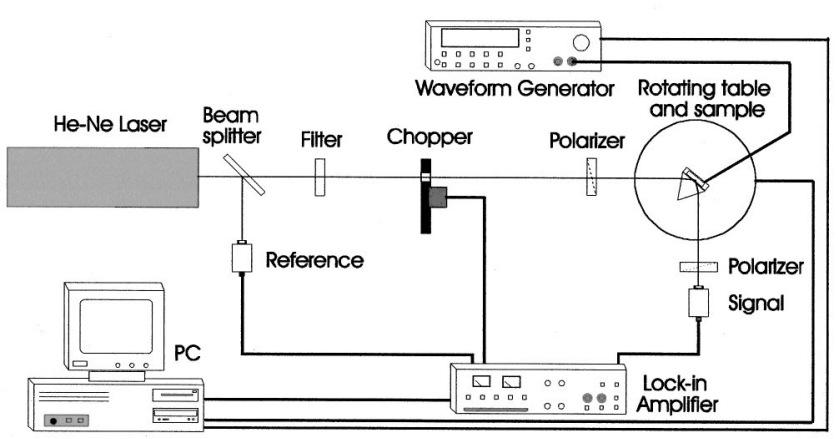

FIG. 1. (a) Cell geometry; the planar side has been chosen as the positive reference. The laser beam impinges from the homeotropic side. Surface polarization vector $\mathbf{P}_{\mathrm{SP}}$ points toward the homeotropic surface, opposite the $z$-axis direction. (b) Half-leaky guided mode experimental setup.

$$
f_{e}=\frac{1}{2}\left[\left(\varepsilon_{\|} \cos ^{2} \theta+\varepsilon_{\perp} \sin ^{2} \theta\right) E^{2}\right]+\mathbf{P}_{\mathrm{SP}} \cdot \mathbf{E}+\mathbf{P}_{f} \cdot \mathbf{E},
$$

where $\mathbf{P}_{\mathrm{SP}}$ is the surface polarization and $\mathbf{P}_{f}$ the flexoelectric polarization [12]. The latter and its $z$ component $P_{z}$ are, respectively,

$$
\begin{gathered}
\mathbf{P}_{f}=e_{1} \mathbf{n}(\boldsymbol{\nabla} \cdot \mathbf{n})+e_{3}(\nabla \times \mathbf{n}) \times \mathbf{n}, \\
P_{z}=-\frac{\left(e_{1}+e_{3}\right)}{2} \sin 2 \theta \frac{d \theta}{d z} .
\end{gathered}
$$

The Euler-Lagrange equation under the constant applied voltage condition [11] leads to
TABLE I. Refractive indices and thickness of ITO, $\mathrm{SiO}_{x}$ substrates, and the LC 5CB layer.

\begin{tabular}{cccc}
\hline \hline & $n_{o}$ & $n_{c}$ & thickness \\
\hline ITO & 1.9 & 1.9 & $100 \mathrm{~nm}$ \\
$\mathrm{SiO}_{x}$ & $\approx 2.0$ & $\approx 1.7$ & $\approx 30 \mathrm{~nm}$ \\
$\mathrm{LC} 5 \mathrm{CB}\left(27^{\circ} \mathrm{C}\right)$ & 1.532 & 1.700 & $8.46 \mu \mathrm{m}$ \\
\hline \hline
\end{tabular}

$$
-\left(\frac{d V}{d z}\right)\left(\varepsilon_{\|} \cos ^{2} \theta+\varepsilon_{\perp} \sin ^{2} \theta\right)+P_{z}+P_{\mathrm{SP}}=D_{z}=C .
$$

$P_{\mathrm{SP}}$ indicates the $z$ component of the surface polarization, and

$$
\begin{gathered}
f(\theta)\left(\frac{d \theta}{d z}\right)^{2}-\frac{D_{z}^{2}}{\left(\varepsilon_{\|} \cos ^{2} \theta+\varepsilon_{\perp} \sin ^{2} \theta\right)} \\
+\frac{P_{z}^{2}}{\left(\varepsilon_{\|} \cos ^{2} \theta+\varepsilon_{\perp} \sin ^{2} \theta\right)}=A .
\end{gathered}
$$

By analogy with the ac voltage case, Eq. (9) and (10) are converted to the corresponding integral equations of voltage $V$ and thickness $d$ :

$$
V=-\int_{0}^{d} \frac{D_{z}-P_{z}}{\left(\varepsilon_{\|} \cos ^{2} \theta+\varepsilon_{\perp} \sin ^{2} \theta\right)} d z+\frac{P_{1}}{\varepsilon_{p}}
$$

where $P_{l}=\int_{0}^{d_{p}} P_{\mathrm{SP}} d z, d_{p}$ is the region of localization of dipoles, and $\varepsilon_{p}$ is the dielectric constant of that region. This last term is different from zero only when the two aligning surfaces are distinct, as in our case [13],

$$
d=\int_{\theta_{1}}^{\theta_{2}} \sqrt{N(\theta)} d \theta
$$

where $\theta_{1}$ is the tilt angle at the homeotropic surface and

$$
N(\theta)=\frac{f(\theta)+\frac{\left(e_{1}+e_{3}\right)^{2} \sin ^{2} \theta \cos ^{2} \theta}{\left(\varepsilon_{\|} \cos ^{2} \theta+\varepsilon_{\perp} \sin ^{2} \theta\right)}}{\frac{D_{z}^{2}}{\left(\varepsilon_{\|} \cos ^{2} \theta+\varepsilon_{\perp} \sin ^{2} \theta\right)}-\frac{D_{z}}{\left(\varepsilon_{\|} \cos ^{2} \theta_{2}+\varepsilon_{\perp} \sin ^{2} \theta_{2}\right)}+f\left(\theta_{2}\right)\left(\frac{d \theta}{d z}\right)_{\theta=\theta_{2}}^{2}+\frac{\left(e_{1}+e_{3}\right)^{2} \sin ^{2} \theta_{2} \cos ^{2} \theta_{2}}{\left(\varepsilon_{\|} \cos ^{2} \theta_{2}+\varepsilon_{\perp} \sin ^{2} \theta_{2}\right)}} .
$$

The tilt angle profile is determined from the solution of Eqs. (11) and (12). We do not take into account any bulk charge distribution as reported in [14] since the low voltages applied and the compensated pulse technique used experimentally do not allow double layer formation, which could drop the voltage over a region of order of the Debye length.

Beside the bulk equations, we have to consider the surface torque balance. While in the ac case it is given by

$$
W \frac{\sin 2 \theta_{2}}{2}-\left(k_{11} \sin ^{2} \theta_{2}+k_{33} \cos ^{2} \theta_{2}\right)\left(\frac{\partial \theta}{\partial z}\right)_{\theta=\theta_{2}}=0
$$

where $W$ is the anchoring energy, for a dc voltage it becomes [4] 

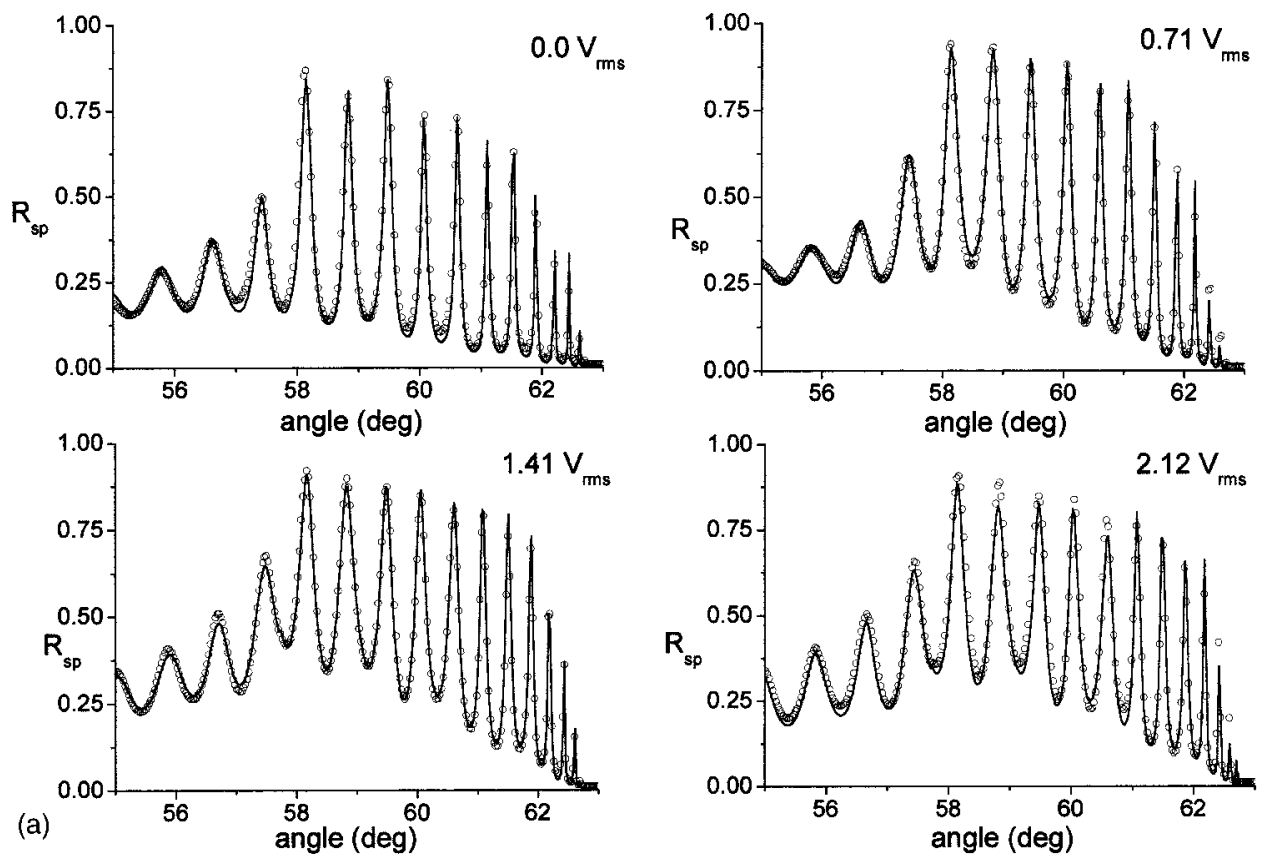

FIG. 2. (a) Fits (lines) of ac voltage reflectivity data (dots). (b) Comparisons of experimental and theoretical tilt angle profiles at 0 , $0.7,1.4$, and $2.1 V_{\mathrm{rm}}$, curves $a-d$, respectively.

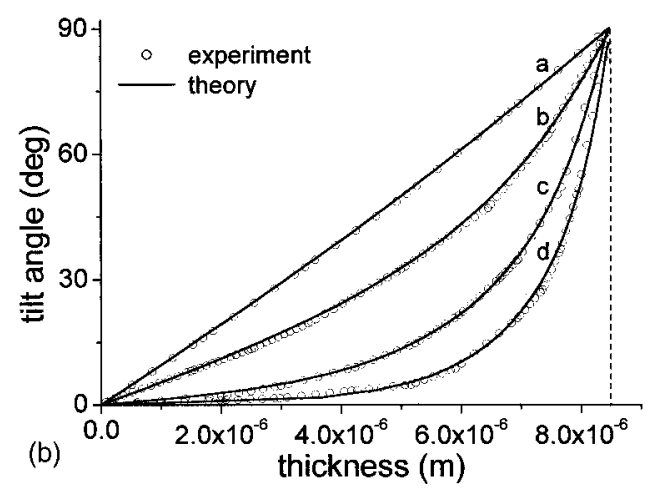

$$
\begin{gathered}
W \frac{\sin 2 \theta_{2}}{2}-\left(k_{11} \sin ^{2} \theta_{2}+k_{33} \cos ^{2} \theta_{2}\right)\left(\frac{\partial \theta}{\partial z}\right)_{\theta=\theta_{2}} \\
-\frac{\left(e_{1}+e_{3}\right)}{2} E\left(\theta_{2}\right) \sin 2 \theta_{2}=0 .
\end{gathered}
$$

\section{EXPERIMENT}

The HLGM optical technique [15] is illustrated in Fig. 1. A thin nematic-liquid-crystal layer is sandwiched between a glass pyramid having a refractive index higher than the largest of the LC and a low refractive index substrate having an index lower than the lowest of the LC. The laser beam impinges from the homeotropic side. This configuration has been chosen because 5CB has positive dielectric anisotropy and an external electric field will change the pretilt angle at the substrate (planar side). The HLGM technique is much more sensitive to changes at this surface.

In this geometry, there exists an angular window from the pseudocritical angle between the high index pyramid and the effective index of the liquid crystal to the critical angle between the high index pyramid and the low index substrate, over which sharp half-leaky resonant guided modes may propagate. For any director twist out of the incidence plane, there is a significant $\operatorname{TM}(p)$ to $\operatorname{TE}(s)$ conversion in the cell. Then in the half-leaky guided wave angle window, the $p$ to $s$ conversion reflectivity gives a series of sharp peaks that are remarkably sensitive to the director profile within the cell. The reflectivity peaks are sharp because the optical field is fully reflected at the liquid-crystal substrate boundary, while being relatively strongly reflected at the high index glassliquid-crystal boundary. Also by measuring the $p$ to $s$ (or $s$ to p) conversion signals that result from the director being out of the plane of incidence, the technique becomes in effect of higher order than measurement of the simple $p$ to $p$ or $s$ to $s$ reflectivity. This thereby allows the detailed characterization of the optical tensor profile in the cell.

In order to obtain the data in the required form of reflectivity from the prism-liquid-crystal boundary as a function of angle of incidence, the complete cell is set on a computercontrolled rotation stage. A He- $\mathrm{Ne}(\lambda=632.8 \mathrm{~nm})$ light beam modulated at $1736 \mathrm{~Hz}$, to allow phase-sensitive detection, impinges on one face of the prism such that it arrives at the liquid-crystal layer at the desired angle of incidence. The incident beam is plane-polarized, either $s$ or $p$, and a second polarizer is placed in front of the detector to give either a $p$ 

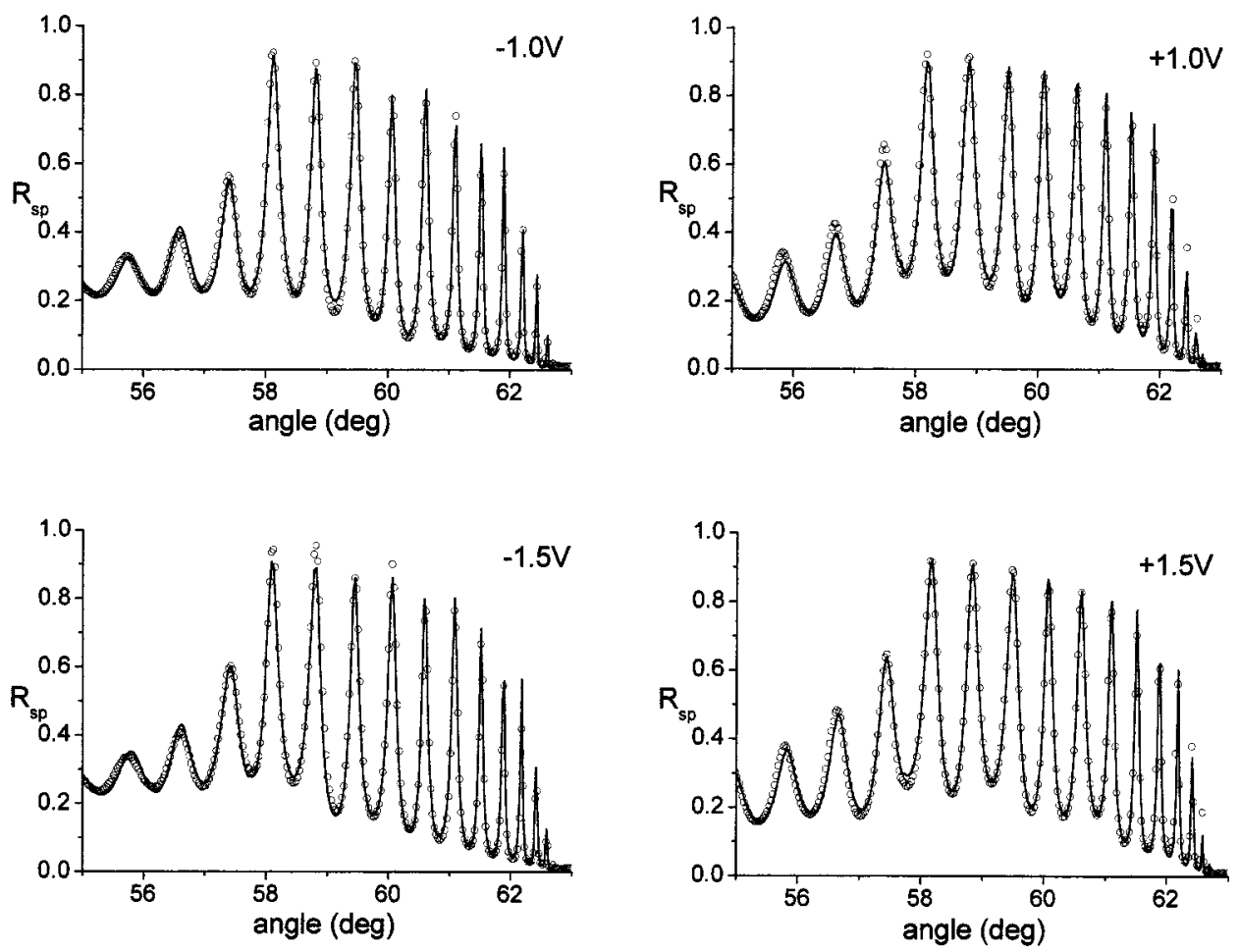

FIG. 3. Fits (lines) of dc voltage reflectivity data (dots). or $s$ signal component. To allow for any variation in laser source intensity, a small reflection is taken from the input beam to act as a reference.

The cell is prepared as in Fig. 1, strong planar anchoring is obtained by deposition of $\mathrm{SiO}_{x}$ at $60^{\circ}$ evaporation angle, while the homeotropic anchoring is provided by octadecyltrimethoxysilane (Fluka), which does not contribute free charges. The LC used is 5CB (Merck); a transparent electrode of indium-tin oxide (ITO), 100-nm nominal thickness, covers the glass plates.

In a hybrid cell, the uniaxial liquid-crystal axis is almost everywhere not normal to the cell walls. We need to fit angle-dependent reflectivity data to Fresnel model predictions based on a reasonable profile of the nematic director. The Fresnel multilayer [16] modeling uses a scattering matrix method [17] with the liquid-crystal layer divided into 50 or more sublayers, depending on the tilt angle gradient. Berreman's $4 \times 4$ matrix technique is used in the modeling. A reasonable hypothesis is first made of the cell optical parameters and the director profile. This produces a model prediction of the angle-dependent reflectivity that is compared with the data. Adjustments are then iteratively made to all the parameters until a minimum least-squares fit to the data is obtained, giving a complete evaluation of the optical structure [18].

Initial measurements are made at temperatures for which the LC is in the isotropic phase in order to evaluate more easily the substrate parameters that are kept fixed for the successive fits. These values are listed in Table I, together with the low-temperature LC indices and the cell thickness.

In all the figures we choose to display $s$ to $p$ reflectivity. The main reason is that this data set, in our experimental conditions, is more sensitive to the director profile than the others. All the figures refer to measurements made with a director twist of $45^{\circ}$ with respect to the incident plane. We also took data for a $0^{\circ}$ twist for which $R_{s p}$ and $R_{p s}$ are zero over the whole angular range.

Measurements have been taken for all the four possible polarization configurations. $R_{p p}, R_{s s}, R_{p s}$, and $R_{s p}$ fittings are undertaken simultaneously for the four data sets to avoid degeneracy of the solutions.

\section{DISCUSSION AND CONCLUSIONS}

ac measurements

Fits of ac $(1 \mathrm{kHz})$ voltage data give tilt angle profiles that match the ones produced by continuum theory, Fig. 2. At 1 $\mathrm{kHz}$ the liquid crystal is in the dielectric regime. Solution of the torque balance equation (13) gives a value for the homogeneous anchoring energy $W=(2.0 \pm 0.1) \times 10^{-4} \mathrm{~J} / \mathrm{m}^{2}$ that is very close to the one obtained elsewhere for the same materials [19]. This value is typical of the quite strong anchoring expected; as a consequence, the variation of the tilt angle at the surface is discernible only for voltages above $\sim 2 V_{\text {rms }}$. In fact, as shown in Fig. 2(b), curves, $a, b$, and $c$ have $90^{\circ}$ pretilt, while curve $d$, for $2.1 V_{\text {rms }}$, has an $86.5^{\circ}$ pretilt.

\section{dc measurements}

For the dc measurements, we apply an alternative squarepulsed signal with zero mean value in order to avoid any long-term cell damage due to electrochemical reaction. The width of the square signal is $1 \mathrm{~s}$, longer than the response time of the liquid crystal. Data are taken only when the optical response has reached the equilibrium value of the particular dc applied voltage. The square signal width has to be smaller than the time that free charges require to screen the 
(a)

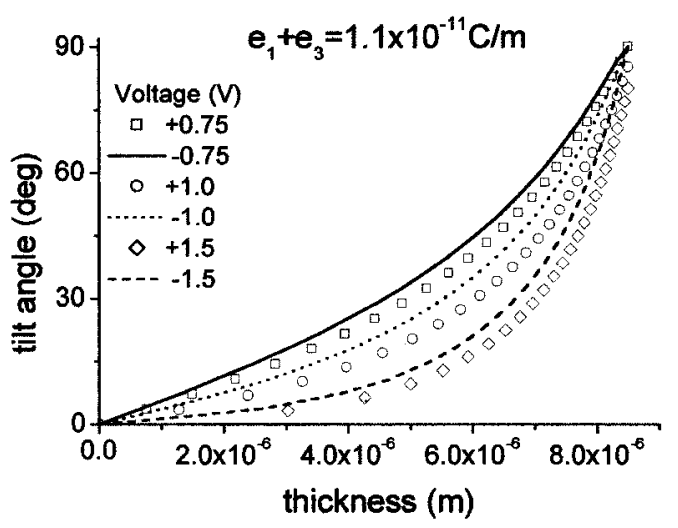

(b)

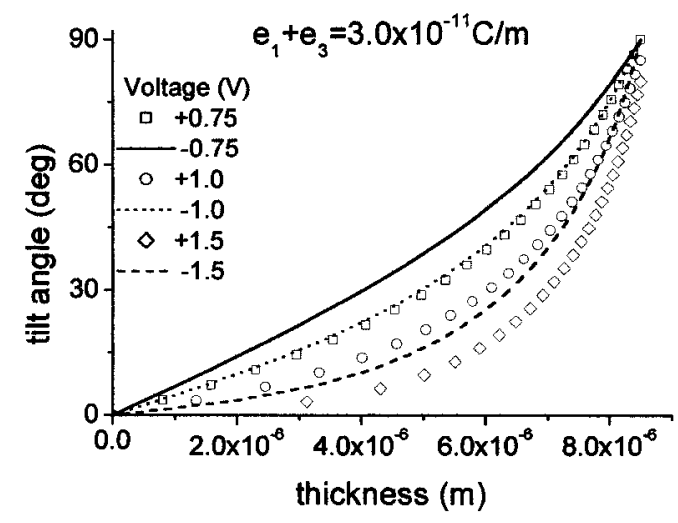

(c)

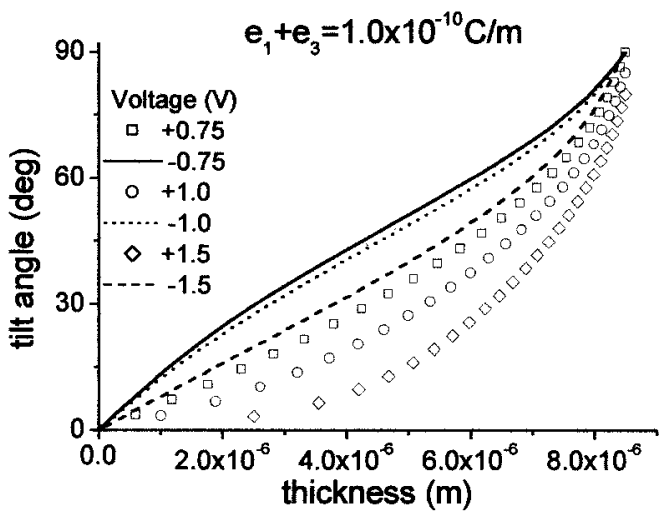

FIG. 4. Theoretical tilt profiles for three values of flexoelectric coefficients at several dc voltages. Surface polarization is not taken into account.

applied field; this is established in our case from measurements of the optical response and impedance measurements (data not shown).

The tilt angle profiles used to fit the reflectivity curves show a remarkable difference between positive and negative polarities (Fig. 3) as well as being different from that obtained for the ac voltages.

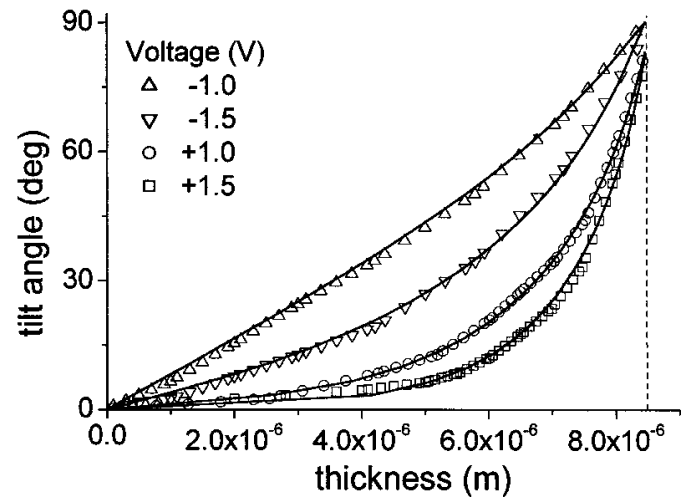

FIG. 5. Comparison between experimental (dots) and theoretical (lines) tilt angle profiles for dc applied voltages. Here surface polarization is included in the model.

The theoretical tilt angle profiles generated including only the flexoelectric term are not able to match the ones used to fit the reflectivity data for any values of flexoelectric coefficients over a reasonable range from $10^{-11}$ to $10^{-10} \mathrm{C} / \mathrm{m}$. In fact, taking low values of the coefficients, the predicted model profiles for opposite polarities at the same voltage are much too similar; see Fig. 4(a). Furthermore, for intermediate values of the coefficients, the difference between profiles of opposite polarity is simply not sufficient to fit the data; see Fig. 4(b). Finally, for high values of the coefficients, profiles of negative polarity at different voltages are almost coincident; see Fig. 4(c).

To overcome this problem, it is necessary to take into account the surface polarization term, $P_{\mathrm{SP}}$ as is included in the theory, Eq. (9). The origin of $P_{\mathrm{SP}}$ may be due to the different affinity of molecules to different substrates, to a spatial dependence of the nematic order parameter close to the interface, and to adsorption of impurities. The vector $\mathbf{P}_{\mathrm{SP}}$ points toward the homeotropic surface (Fig. 1), parallel to the flexoelectric polarization; from the sign found, it is reasonable to think that this term arises through preferential adsorption of ions at one of the two surfaces $\left(\mathrm{SiO}_{x}\right)$, thereby polarizing it.

The contributions to the energy of the liquid crystal due to polarization are $-\mathbf{P}_{f} \cdot \mathbf{E}-\mathbf{P}_{\mathrm{SP}} \cdot \mathbf{E}-\mathbf{P}_{f} \cdot \mathbf{E}_{S}$. The first represents the coupling between the flexoelectricity and the external field, the second the coupling between the surface polarization and the external field, and the third the coupling between flexoelectricity and the surface field.

The effect of the term $-\mathbf{P}_{\mathrm{SP}} \cdot \mathbf{E}$ enhances the tilt profile distortion for positive voltages, while for negative voltages it reduces the effective field in the cell; in other words, it produces a voltage bias [see Eq. (11)]. The last term, $-\mathbf{P}_{f} \cdot \mathbf{E}_{S}$, should influence the director profile even at zero applied voltage and at ac applied voltages but, since at ac the experimental profiles match the theoretical ones [Eqs. (4) and (5)], this term can be neglected. The surface field $\mathbf{E}_{s}$ is effectively screened by the surface counterions. No substantial field penetration into the bulk is expected and thus the term should be considered negligible in the volume equation $[20,21]$. 
It is important to stress that once $P_{\mathrm{SP}}$ is added to the model, a flexoelectric coefficient of $\left(e_{1}+e_{3}\right)=3$ $\times 10^{-11} \mathrm{C} / \mathrm{m}$ is able to reproduce the experimental tilt angle profiles; see Fig. 5. Note that, in this figure, curves for negative voltage have $90^{\circ}$ pretilt, while the $+1.0-\mathrm{V}$ curve has $83^{\circ}$ and the $+1.5-\mathrm{V}$ curve has $82^{\circ}$.

The value of $P_{l}$ Eq. (11), required to fit the data is about $10^{-11} \mathrm{C} / \mathrm{m}$. This corresponds to a surface polarization $P_{\mathrm{SP}}$ $\sim 3 \times 10^{-4} \mathrm{C} / \mathrm{m}^{2}$ taking the dipole length to be the $\mathrm{SiO}_{x}$ layer thickness (otherwise $P_{\mathrm{SP}} \sim 3 \times 10^{-3} \mathrm{C} / \mathrm{m}^{2}$ if the dipole length is that of the $\mathrm{SiO}_{x}$ molecule). The corresponding number of electrons per $\mathrm{SiO}_{x}$ molecule is $2 \times 10^{-6}$ if we take the $\mathrm{SiO}_{x}$ layer as the dipole or $2 \times 10^{-4} e^{-} / \mathrm{mol}$ in the case of the molecular dipole.

The energy associated with the surface polarization is $-\mathbf{P}_{\mathrm{SP}} \cdot \mathbf{E} \sim 2 \times 10^{-6} \mathrm{~J} / \mathrm{m}^{2}$ at the highest voltage applied, the same order of value found in [14] for a well-ordered layer of molecules on the surface. The energy evaluation in [14] is made considering a dipole moment of well-ordered LC molecules at the surface, but as reported in [22], "a first layer of $\mathrm{LC}$ molecules is strongly adsorbed at the surface of $\mathrm{SiO}_{x}$ and it cannot reorient even under very strong torque," and the "adsorbed layer becomes a part of the substrate."

The above observation supports the idea of the $\mathrm{SiO}_{x}$ layer polarization. The value of $P_{l}$ found in our case is low compared to the one reported in [22], where the same system has been studied. In that work, the authors consider a layer of adsorbed LC molecules having a certain angle from the normal, $P_{l}^{\prime}=P_{l} \cos \theta$, hence the value of $P_{l}$ is higher (for example, if $\theta=80^{\circ}$, the corresponding polarization is $\left.5 \times 10^{-11} \mathrm{C} / \mathrm{m}\right)$.

In conclusion, we deduce that even if the surface polarization energy is low compared to the anchoring energy, the field and the shift of the potential due to the dipoles at the surface strongly affect the profiles. Our model, even if approximate, gives a reasonable value of the flexoelectric coefficient and surface polarization, comparable to values found in the literature. Furthermore, this technique gives information on the director profile and allows an independent determination of the two contributions. This feature is very important since integrated techniques allow only the determination of the sum of these terms [2-9]. We do not understand the discrepancy of the sign of the flexoelectric coefficient with Refs. [8,9], where a pyroelectric method is used.

\section{ACKNOWLEDGMENTS}

The authors thank A. Scarfato for the $\mathrm{SiO}_{x}$ evaporation, B. De Nardo, C. Prete, and M. Sposato for help building the setup, and Professor R. Bartolino. The authors also appreciate the support of the Thematic Network "Optical Research of Chiral Systems," EC Program Human Capital and Mobility No. ERB FMRX-CT97-0119.
[1] R. B. Meyer, Phys. Rev. Lett. 22, 918 (1968).

[2] I. Dozov, Ph. Martinot-Lagarde, and G. Durand, J. Phys. (France) Lett. 43L, 365 (1982).

[3] P. R. Maheswara Murthy, V. A. Raghunathan, and N. V. Madhusudana, Liq. Cryst. 14, 483 (1993).

[4] S. R. Warrier and N. V. Madhusudana, J. Phys. II 7, 1789 (1997).

[5] J. P. Marcerou and J. Prost, Mol. Cryst. Liq. Cryst. 58, 259 (1980)

[6] N. V. Madhusudana and G. Durand, J. Phys. (France) Lett. 46L, 195 (1985).

[7] L. A. Beresnev et al., Pis'ma Zh. Eksp. Teor. Fiz. 45, 592 (1987) [JETP Lett. 45, 755 (1987)].

[8] L. M. Blinov, M. Ozaki, and K. Ioshino, Pis'ma Zh. Eksp. Teor. Fiz. 69, 220 (1999) [JETP Lett. 69, 236 (1999)].

[9] L. M. Blinov, M. I. Barnik, M. Ozaki, N. M. Shtykov, and K. Yoshino, Phys. Rev. E 62, 8091 (2000).

[10] C.-J. Chen, A. Lien, and M. I. Nathan, J. Appl. Phys. 81, 70 (1997).

[11] R. N. Thurston and D. W. Berreman, J. Appl. Phys. 52, 508
(1981).

[12] H. J. Deuling, Solid State Phys. 14, 77 (1978).

[13] V. G. Nazarenko, R. Klouda, and O. D. Lavrentovich, Phys. Rev. E 57, 36 (1998).

[14] S. Ponti, P. Ziherl, C. Ferrero, and S. Zumer, Liq. Cryst. 26, 1171 (1999).

[15] F. Yang and J. R. Sambles, J. Opt. Soc. Am. B 10, 858 (1993).

[16] R. M. A. Azzam and N. M. Bashara, Ellipsometry and Polarised Light (North-Holland, Amsterdam, 1979).

[17] D. Y. K. Ko and J. R. Sambles, J. Opt. Soc. Am. A 5, 1863 (1988).

[18] F. Yang and J. R. Sambles, Mol. Cryst. Liq. Cryst. 50, 143 (1994).

[19] K. R. Welford and J. R. Sambles, Appl. Phys. Lett. 50, 871 (1987).

[20] R. Meister and B. Jerome, J. Appl. Phys. 86, 2473 (1999).

[21] U. Kuhnau, A. G. Petrov, G. Klose, and H. Schmiedel, Phys. Rev. E 59, 578 (1999).

[22] S. Forget, I. Dozov, and Ph. Martinot-Lagarde, Mol. Cryst. Liq. Cryst. Sci. Technol., Sect. A 329, 605 (1999). 